\title{
HABILIDADES DE COMUNICACIÓN DURANTE LA ENTREVISTA CLÍNICA: SIMULACIÓN DEL PACIENTE Y ROLE PLAYING ${ }^{1}$
}

\author{
COMMUNICATION SKILLS DURING THE CLINICAL INTERVIEW: \\ SIMULATED PATIENT AND ROLE PLAYING
}

\section{HABILIDADES DE COMUNICAÇÃO DURANTE A ENTREVISTA CLÍNICA: SIMULAÇÃO DO PACIENTE E ROLE PLAYING}

\author{
Patricia Rojas Santibáñez* \\ Sara Guerrero Núñez** \\ Cristina Arancibia Gajardo*** \\ Macarena BarRaZa NúŃEZ $Z^{* * * *}$
}

\begin{abstract}
RESUMEN
Objetivo: Evaluar las habilidades de comunicación durante la entrevista clínica, en el contexto de la Simulación Clínica (SC) con Paciente Simulado (PS) y Role Playing (RP). Material y Método: Investigación con aplicación del método mixto de tipo secuencial explicativo, en el contexto de una asignatura electiva de innovación en el Departamento de Enfermería de la Universidad de Atacama. La primera etapa fue cuantitativa de tipo correlacional, aplicando una versión abreviada de la Master Interview Rating Scale (MIRS) a diez estudiantes de enfermería. Cada uno realizó tres entrevistas clínicas con metodología de SC con PS, y tres con RP. Para la hipótesis correlacional se calculó $t$ student. La segunda etapa fue cualitativa de tipo fenomenológica, alcanzando la saturación con dos grupos focales. Los datos se analizaron línea por línea, con categorización emergente, y triangulación de investigadores y datos. Resultados: Se obtuvo un $t$ student de 12,161, aceptando la hipótesis: "En la SC con PS los estudiantes demuestran una mejor aplicación de las habilidades de comunicación”. Se identificaron 23 códigos y tres categorías centrales que describen la importancia de la SC en la aplicación de las habilidades de comunicación. Conclusiones: La aplicación de habilidades de comunicación durante la entrevista clínica fue evaluada positivamente desde la dimensión cuantitativa y cualitativa, obteniendo puntuaciones que
\end{abstract}

${ }^{1}$ Proyecto financiado por proyecto DIUDA de la Universidad de Atacama.

*Enfermera-Matrona, Magíster en Docencia para la Educación Superior, Departamento de Enfermería, Facultad de Ciencias de la Salud, Universidad de Atacama, Copiapó, Chile. ORCID: https://orcid.org/0000-0002-2591-6045 Email: patricia.rojas@uda. cl. Autora de correspondencia.

**Enfermera, Doctora en Enfermería, Departamento de Enfermería, Facultad de Ciencias de la Salud, Universidad de Atacama, Copiapó, Chile. ORCID: https://orcid.org/0000-0001-5004-3760 Email: sara.guerrero@uda.cl. Autora de correspondencia.

***Enfermera, Magíster en Gestión en Atención Primaria de Salud, Departamento de Enfermería, Facultad de Ciencias de la Salud, Universidad de Atacama, Copiapó, Chile. ORCID: https://orcid.org/0000-0002-7062-1449 Email: cristina.arancibia@ uda.cl

${ }^{* * * *}$ Enfermera, Magíster en Docencia para la Educación Superior, Departamento de Enfermería, Facultad de Ciencias de la Salud, Universidad de Atacama, Copiapó, Chile. ORCID: https://orcid.org/0000-0003-3170-8426 Email: macarena.barraza@uda.cl 
demuestran un alto porcentaje de cumplimiento y una evaluación perceptiva positiva entre los participantes.

Palabras clave: Habilidad; Comunicación; Entrevista; Simulación de paciente; Role Playing; Master Interview Rating Scale.

\begin{abstract}
Objective: To evaluate communication skills during the clinical interview, in the context of Clinical Simulation (CS) with Simulated Patient (SP) and Role Playing (RP). Material and Method: Application research using sequential explanatory mixed method in the context of an optional innovation course at the Department of Nursing of the University of Atacama (Chile). The first stage was quantitative correlational, applying an abbreviated version of the Master Interview Rating Scale (MIRS) to ten nursing students. Each one conducted three clinical interviews using CS methodology with SP, and three with RP. For the correlational hypothesis, Student's $t$ was calculated. The second stage was qualitative phenomenological, reaching saturation with two focus groups. The data was analyzed line by line, with emergent categorization, and with triangulation of researchers and data. Results: A Student's $t$ of 12,161 was obtained, accepting the hypothesis: "In the CS with SP students demonstrate a better application of communication skills". 23 codes and 3 central categories that describe the importance of the CS in the application of communication skills were identified. Conclusions: The application of communication skills during the clinical interview was positively evaluated from the quantitative and qualitative dimensions; obtaining scores that show a high percentage of compliance, and a positive perceptual evaluation among participants.
\end{abstract}

Key words: Skill; Communication; Interview; Patient Simulation; Role Playing; Master Interview Rating Scale.

\title{
RESUMO
}

Objetivo: Avaliar as habilidades de comunicação durante a entrevista clínica, no contexto de Simulação Clínica (SC) com Paciente Simulado (PS) e Role Playing $(R P)$. Material e Método: Pesquisa com aplicação do método sequencial misto, no contexto de uma disciplina de inovação eletiva do Departamento de Enfermagem da Universidade de Atacama (Chile). A primeira etapa foi quantitativa do tipo correlacional, aplicando-se uma versão abreviada da Master Interview Rating Scale (MIRS) a dez estudantes de enfermagem. Cada um realizou três entrevistas clínicas com a metodologia SC com PS e três com RP. Para a hipótese correlacional, foi calculado o $t$ de Student. A segunda etapa foi qualitativa de tipo fenomenológico, alcançando a saturação com dois grupos focais. Os dados foram analisados linha a linha, com categorização emergente, e com triangulação de pesquisadores e dados. Resultados: Obteve-se um $t$ de Student de 12.161, aceitando a hipótese: "Em SC com PS, os alunos demonstram uma melhor aplicação das habilidades de comunicação". Foram identificados 23 códigos e três categorias centrais que descrevem a importância da SC na aplicação das habilidades de comunicação. Conclusôes: A aplicação das habilidades de comunicação durante a entrevista clínica foi avaliada positivamente na dimensão quantitativa e qualitativa; obtenção de escores que mostram alto percentual de adesão e avaliação perceptual positiva entre os participantes.

Palavras-chave: Habilidade; Comunicação; Entrevista; Simulação de paciente; Role Playing; Master Interview Rating Scale.

Fecha de recepción: 30/11/2020

Fecha de aceptación: 13/7/2021 


\section{INTRODUCCIÓN}

El actual modelo educativo de enseñanza universitaria promueve la adquisición de competencias necesarias para el desarrollo profesional, destacándose la comunicación oral entre las competencias genéricas ${ }^{(1)}$. La comunicación es fundamental para establecer una buena relación terapéutica, promoviendo en los pacientes la restauración de la salud $^{(2)}$. La comunicación eficaz entre el profesional y paciente influye en la experiencia de este último, mejorando la comprensión del estado de salud, el tratamiento y cuidado. En consecuencia, la comunicación eficaz es una métrica importante en las instituciones de salud, dada su contribución directa en los indicadores sanitarios. Por lo anterior, la comunicación es vital en la formación de los profesionales de la salud, especialmente en enfermeros(as), quienes proporcionan un alto porcentaje de atenciones de salud, enfrentando situaciones complejas que demandan empatía, respeto, claridad en el lenguaje, selección del momento oportuno, y entre otros, escucha activa ${ }^{(3)}$.

La entrevista clínica es una actividad habitual en la atención de salud y demanda conocimientos, actitudes y habilidades en el profesional de enfermería. La habilidad de comunicación es fundamental, pues le permite "obtener información específica y necesaria para el diagnóstico de enfermería y la planificación de los cuidados, además de facilitar la relación enfermera/paciente [...] que favorecería la prestación de una atención individualizada de mayor calidad"(4). Por esto es necesario promover una entrevista clínica de calidad, incorporando metodologías que incrementen la aplicación de habilidades de comunicación durante la transición de estudiante a profesional. Dicha transición puede ser comprendida desde el modelo de adquisición de habilidades y competencias de los hermanos Dreyfus ${ }^{(5)}$, que clasifica los aprendizajes en principiante, principiante avanzado, competente, eficiente y experto. Estos han sido estudiados y aplicados en la filosofía de Patricia Benner $^{(6)}$, permitiendo identificar necesidades de aprendizajes durante la formación de enfermeros(as), y comprender la adquisición de competencias por medio de la simulación clínica ${ }^{(7)}$. Esta es una metodología activa e innovadora, que a través de experiencias guiadas replican un entorno real $^{(8,9)}$, generando un aprendizaje por medio del error, sin que esto ocasione consecuencias directas en el paciente. Esto último es primordial al considerar que cada año millones de personas sufren daño por causa de una atención sanitaria. Entre las principales estrategias de SC se encuentra el PS, que corresponde a "una persona que ha sido entrenada cuidadosamente para simular a un paciente real"(10). El $R P$ es otra estrategia, donde los estudiantes "asumen con autonomía el papel del paciente o profesional, ante una situación simulada" ${ }^{(11)}$.

La práctica del cuidado supone un saber experto alcanzado por medio de la formación académica y experiencias clínicas previas, y para ello el estudiante debe transitar en un aprendizaje que le permita adquirir competencias. Teniendo presente que la SC ha demostrado beneficios en la transición de un saber, saber hacer y saber ser, en el ámbito de las habilidades de comunicación, tanto en programas de pregrado, posgrado y especialidades, y considerando que es fundamental estudiarla en dicho contexto para la evaluación y actualización de los currículums, este estudio tuvo como objetivo evaluar las habilidades de comunicación durante la entrevista clínica, en el contexto de la SC con PS y $R P$.

El estudio se realizó en un grupo de estudiantes que aprobaron la primera asignatura práctica (principiantes o novatos en habilidades de comunicación para entrevista clínica), y quienes integraron un electivo dirigido a generar competencias comunicacionales durante la entrevista clínica.

\section{MATERIAL Y MÉTODO}

Investigación mixta con diseño secuencial explicativo, considerando dos principales etapas en el proceso investigativo (CUAN-CUAL). Este diseño permitió desarrollar una explicación secuencial y más acabada del fenómeno de estudio, con una integración de datos durante la discusión, vinculando los resultados obtenidos en ambas etapas. El proyecto fue aprobado por la Comisión de Investigación de la institución y ejecutado durante el primer semestre del año 2019, en el contexto de una asignatura electiva de innovación denominada "Entrevista Clínica”. Esta fue diseñada para desarrollar habilidades de comunicación durante la entrevista clínica, a través de la estrategia 
metodológica de SC con PS y $R P$, preparando al estudiante para futuras prácticas clínicas, especialmente las de mayor complejidad.

Los estudiantes fueron preparados para enfrentar una entrevista clínica de calidad, desde el saber, saber ser y saber hacer. El estudio consideró al universo de estudiantes inscritos en el electivo (10 participantes del tercer año en enfermería), porque el criterio de inclusión consideró a estudiantes en el primer nivel de aprendizaje, según la filosofía de Benner $^{(6)}$ : novato o principiante, es decir, quienes aprobaron la primera asignatura práctica y sin previa experiencia en SC. Esta investigación se realizó al término del electivo, en la sala espejo de la unidad académica y posterior a la firma del consentimiento informado.

La versión original de la Master Interview Rating Scale (MIRS) contiene veinte y ocho dimensiones o criterios, con respuestas puntuadas de 1 a 5 en escala tipo Likert ${ }^{(12)}$. En la investigación se aplicó una versión abreviada de la escala en español del año 2012 aplicada en Chile ${ }^{(13)}$, evaluando catorce dimensiones seleccionadas por las tres investigadoras del proyecto (Tabla 1).

El análisis de competencias se logró dado el perfil de las investigadoras: enfermeras con una vasta experiencia clínica (promedio de 20 años), larga trayectoria en docencia (promedio de 12 años), dos con postítulos y posgrados en docencia para la educación superior, dos diplomadas en simulación clínica y una instructora en simulación clínica.

Previo a la investigación, y para resguardar la fiabilidad del instrumento, se evaluó la concordancia inter-observador por medio del índice Kappa de Fleiss. Para ello las tres investigadoras aplicaron la escala MIRS abreviada en 18 entrevistas clínicas con metodología de SC con PS, realizadas por 18 estudiantes de tercer a quinto año de la carrera de Enfermería, quienes respondieron voluntariamente a una invitación abierta de participación en esos niveles, además de firmar el consentimiento informado. Las investigadoras evaluaron simultáneamente las habilidades de comunicación en la sala espejo.

La primera etapa de investigación fue cuantitativa de tipo correlacional, evaluando las habilidades de comunicación durante la entrevista clínica, a través de la versión abreviada de la escala MIRS. Los diez participantes realizaron la entrevista clínica en seis escenarios confeccionados por las investigadoras, en función de temáticas de actualidad, obteniendo un total de sesenta evaluaciones; treinta en SC con PS y treinta en $R P$. Los escenarios se desarrollaron en las mismas condiciones de ambiente, horario y tiempo, y la SC realizada en la asignatura se estructuró según lo observado en la Figura 1.

Tabla 1. Coeficiente de Kappa de Fleiss en la versión abreviada de la escala MIRS. Copiapó, Región de Atacama, Chile, 2018.

\begin{tabular}{l|l|l}
\hline Ítems o Dimensión & Kappa Fleiss & Clasificación \\
\hline 1. Presentación & 0,947 & Muy buena \\
\hline 2. Exploración de preocupaciones & 0,716 & Buena \\
\hline 4. Hilo Narrativo & 0,613 & Buena \\
\hline 7. Transiciones & 0,838 & Muy buena \\
\hline 9. Tipos de preguntas & 0,621 & Buena \\
\hline 12. No uso de lenguaje médico & 0,756 & Buena \\
\hline 13. Verificación de la información & 0,783 & Buena \\
\hline 15. Técnicas de facilitación verbal & 0,724 & Buena \\
\hline 16. Técnica de facilitación no verbal & 0,746 & Buena \\
\hline 17. Empatía & 0,716 & Buena \\
\hline 19. Impacto de la enfermedad en la autoimagen & 0,801 & Buena \\
\hline 20. Impacto de la enfermedad en la familia. & 0,800 & Buena \\
\hline 27. Invitar a preguntar & 0,739 & Buena \\
\hline 28. Cierre & 0,883 & Muy buena \\
\hline
\end{tabular}



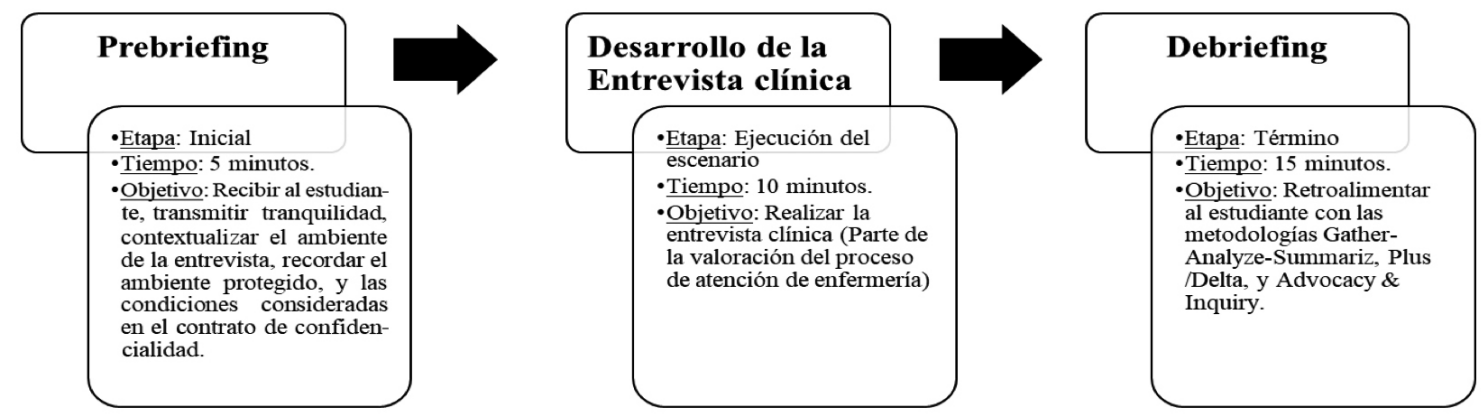

Figura 1. Proceso de la Simulación clínica en la asignatura "Entrevista Clínica”. Copiapó, Región de Atacama, Chile, 2019.

El análisis de la primera etapa considera una estadística descriptiva y la aplicación del estadígrafo $t$ de student, con un nivel de confianza del 95\%, probando la hipótesis de investigación: "En la SC con PS los estudiantes demuestran una mejor aplicación de las habilidades de comunicación".

La segunda etapa de investigación fue cualitativa de tipo fenomenológica, aplicando la filosofía de Patricia Benner ${ }^{(6)}$ en la recolección y análisis de datos. Se realizaron dos grupos focales, de 10 participantes o entrevistados, con una pauta de entrevista dirigida a recoger discursos evaluativos sobre la aplicación de las habilidades comunicativas en ambas estrategias metodológicas: de 60 y 40 min, alcanzando con ellos la saturación de los datos. Además, se aplicaron los criterios de rigor de credibilidad y auditabilidad; realizando una transcripción fidedigna de las grabaciones de voz y notas de campo de ambos grupos focales, y un análisis manual, línea por línea, con categorización emergente, y efectuando triangulación de datos e investigadores (Figura 2). Los entrevistados fueron enumerados del 1 al 10, utilizando la siguiente nomenclatura en las citas textuales: E1, E2, E3.... E10.

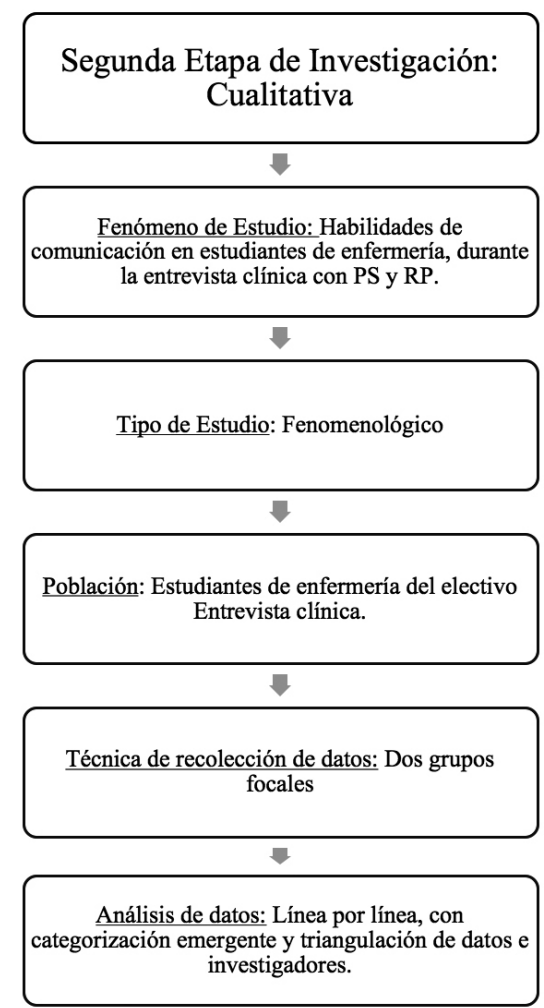

Figura 2. Proceso investigativo. Copiapó, Región de Atacama, Chile, 2019. 


\section{RESULTADOS}

El universo del estudio estuvo constituido por 10 estudiantes: nueve mujeres y un hombre, entre 19 y 28 ańos, todos cursando el tercer ańo (sexto semestre) de la carrera de Enfermería, con aprobación de su primera práctica, y finalizando el electivo de entrevista clínica. Paralelamente los participantes estaban cursando la segunda práctica clínica: médico-quirúrgico de baja y mediana complejidad.

La Tabla 1 evidencia un coeficiente Kappa de Fleiss superior a 0,61 en las catorce dimensiones evaluadas, reflejando una buena fuerza de concordancia, y un buen nivel de confiabilidad en la aplicación de la MIRS por las investigadoras del proyecto. En consecuencia, los resultados permitieron la aplicación de la MIRS en todas las dimensiones evaluadas.

Primera etapa de investigación: Las dimensiones con mayor puntaje en las entrevistas con PS corresponden a "presentación"; con una media de 4,7 puntos, y "técnicas de facilitación no verbal" con una media de 4,56 puntos. Las dimensiones con mayor puntaje en las entrevistas con $R P$ corresponden a "uso de lenguaje técnico" con una media de 4,86 puntos, y "presentación" con una media de 3,9 puntos.

Por otra parte, las dimensiones con menor puntaje en las entrevistas con PS corresponden a "transiciones", con una media de 2,73 puntos, e "invitación a preguntar", con una media de 2,43 puntos. De igual forma, en las entrevistas con $R P$ se observaron las mismas dimensiones con menor puntaje, a saber: "transiciones" e "invitación a preguntar", con una media de 1,2 puntos y 1,36 puntos, respectivamente.

La Tabla 2 expone los puntajes totales obtenidos en la MIRS, los cuales evalúan en su conjunto la aplicación de las habilidades de comunicación en SC con PS y RP. Se obtuvo una media de 56 puntos ( $80 \%$ del puntaje total) en las evaluaciones de SC con PS, y de 44 puntos (63\% del puntaje total) en $R P$, con una diferencia estadísticamente significativa $(t$ student $=12,161)$ que permitió aceptar la hipótesis: En la SC con PS los estudiantes demuestran una mejor aplicación de las habilidades de comunicación.

Para desarrollar una explicación del fenómeno estudiado se exploraron las experiencias de los participantes, analizando cualitativamente los discursos referentes a una evaluación de los estudiantes sobre la aplicación de estas habilidades en los escenarios simulados.

Segunda etapa de investigación: Del análisis emergieron cinco códigos comunes a las metodologías de SC con PS y $R P$, y 18 códigos diferenciados que distinguen la contribución de cada metodología en la aplicación de las habilidades estudiadas. Emergieron tres categorías que abordan la evaluación perceptiva de los estudiantes sobre la aplicación de las habilidades de comunicación en ambas metodologías de SC:

Tabla 2a. Distribución de puntajes en la versión abreviada de MIRS para evaluaciones con metodología de Paciente Simulado y Role Playing. Copiapó, Región de Atacama, Chile, 2019.

\begin{tabular}{|c|c|c|c|c|c|c|c|c|c|c|c|c|c|c|c|}
\hline \multirow[b]{3}{*}{ Puntaje PS } & \multicolumn{15}{|c|}{ Evaluaciones } \\
\hline & 1 & 2 & 3 & 4 & 5 & 6 & 7 & 8 & 9 & 10 & 11 & 12 & 13 & 14 & 15 \\
\hline & 50 & 43 & 48 & 52 & 52 & 55 & 63 & 50 & 60 & 54 & 52 & 46 & 58 & 55 & 51 \\
\hline Puntaje RP & 27 & 39 & 37 & 46 & 37 & 41 & 55 & 48 & 50 & 42 & 43 & 33 & 48 & 43 & 43 \\
\hline
\end{tabular}

Tabla 2 b.

\begin{tabular}{|c|c|c|c|c|c|c|c|c|c|c|c|c|c|c|c|c|}
\hline & \multicolumn{15}{|c|}{ Evaluaciones } & \multirow[t]{2}{*}{ Medias } \\
\hline & 16 & 17 & 18 & 19 & 20 & 21 & 22 & 23 & 24 & 25 & 26 & 27 & 28 & 29 & 30 & \\
\hline Puntaje PS & 54 & 57 & 62 & 60 & 50 & 52 & 60 & 61 & 63 & 63 & 57 & 61 & 63 & 62 & 54 & 56 \\
\hline Puntaje RP & 55 & 45 & 47 & 46 & 43 & 36 & 44 & 48 & 45 & 44 & 43 & 53 & 54 & 42 & 39 & 44 \\
\hline
\end{tabular}




\section{Existe una percepción de realismo en ambas} metodologías de SC (PS y $R \boldsymbol{P})$ : Los estudiantes valoran esta metodología como preparatoria para la experiencia práctica, especialmente por el realismo que experimentan. Aunque ambas metodologías permiten la aplicación de conocimientos y habilidades, la entrevista con PS es percibida como la metodología con mayor realismo, debido al escenario y dinámica semejante a la práctica clínica. En consecuencia, la percepción de realismo contribuye a la transición descrita por Benner ${ }^{(6)}$, favoreciendo así la aplicación de habilidades de comunicación:

"Y en la última me olvidé de la cámara, que estaban ustedes al otro lado, y sentí como que estaba en un box. La última fue muy real, senti que apliqué todo. Fue raro, en verdad me introducía tanto en el tema, que sentia que estaba en un box, y estábamos las dos no más" (E 4). "Sí, me sentía nerviosa, pero como lo sentía a la vez real, trataba de controlarme y enfocarme, porque es un paciente que está conmigo y asi lo sentía" (E 7). "También el hecho que se formó un ambiente mucho más realista, como el tema del maquillaje que fue como bien interesante" (E 4).

2. Los beneficios de la SC con PS se perciben como los atributos que permiten la aplicación de habilidades de comunicación. Estos beneficios contribuyen a la contención de emociones expresadas por el usuario y el cuidado diferenciado acorde a las necesidades detectadas. Estos escenarios metodológicos se califican como significativos para el aprendizaje, pues preparan al estudiante en la comunicación efectiva que debe tener con el paciente, gestionando las emociones, los problemas, o cualquier otra situación que surge en la práctica clínica; así el estudiante transita en la adquisición de saberes que lo convertirán algún día en experto. Los discursos refuerzan los puntajes generales obtenidos en la MIRS, al percibir que la SC con PS es una metodología que permite una mejor aplicación de las habilidades en estudio:

"Yo senti que me sentía más cómoda con mis compañeros, igual recolecté mayor información ahi, pero siento que me beneficié más del paciente simulado, por el hecho de ponerme en aprietos. Era como más realista, trataba de hacerlo mejor" (E 2). "Nos puso en aprietos, eso es lo que ocurre en la realidad. Porque siempre va a ser una persona y un caso nuevo, y no va a ser igual al anterior...varias cosas me han quedado" (E 7). "Siento que me sirvió más el paciente simulado, porque como decian mis compañeros, era como un reto, alguien que nunca habiamos visto, el abrirse para poder conectar con la persona, para conversar y para que ella pueda sentir la confianza para contar" (E 9).

3. Los beneficios de la SC con RP son los atributos percibidos que permiten la aplicación de habilidades de comunicación. Los estudiantes consideran que una aplicación temprana de esta metodología genera mayor efectividad en el aprendizaje y la recomiendan como etapa preparatoria para la SC con PS, especialmente cuando se realiza con desconocidos (compañeros de otros niveles). Considerando la transición de aprendizajes y competencias descritos por Benner ${ }^{(6)}$, los discursos proponen que el proceso formativo diseñado en el currículo incorpore el $R P$ como estrategia metodológica previa al PS, es decir, en asignaturas básicas:

"Porque uno va como adquiriendo más herramientas y después sirven por ejemplo cuando ya llegó la actriz, más o menos sabia cómo actuar, aunque no la conocía, sabia cómo seguir los pasos" (E 1). "Nos serviría, o es beneficioso como etapa preentrenamiento, antes de entrar a la etapa de la actriz" (E 4). "Igual siento que fue más fácil cuando hice entrevistas con mis compañeros...era como que lo hubiera hecho toda la vida" (E 5).

\section{DISCUSIÓN}

Los resultados del estudio realzan, en su conjunto, la importancia de la SC en la aplicación de habilidades comunicativas, siendo esto coherente a lo señalado por algunos autores ${ }^{(14)}$ que identificaron los beneficios percibidos de estudiantes y profesionales sobre la SC con dramatización; el beneficio con mayor frecuencia de aparición fue el de "habilidades y competencias de comunicación”.

Los resultados cuantitativos demuestran que en la estrategia metodológica del PS es más efectiva la aplicación de habilidades comunicativas, semejante a los resultados obtenidos en un estudio realizado en $2019^{(15)}$, donde se compararon el PS y RP en tres dominios: comunicación genérica, comunicación clínica y física, y habilidades en examen o proce- 
dimiento. La SC con PS obtuvo mejores resultados en los tres dominios.

Por otra parte, la evaluación cualitativa sobre la aplicación de dichas habilidades fue positiva en ambos escenarios simulados. Considerando la perspectiva filosófica de Patricia Benner ${ }^{(6)}$, los participantes valoraron la contribución de la SC en la transición de su aprendizaje, desde estudiante novato o principiante a estudiante experto en habilidades de comunicación para la entrevista clínica. En esta misma transición proponen al $R P$ como una estrategia inicial y útil para desarrollar y aplicar habilidades de comunicación menos complejas. Perciben que el transitar a un estado experto en las habilidades comunicativas, necesario en prácticas clínicas más avanzadas, lo alcanzan mayormente por medio de la SC con PS. Aun con esto, autores ${ }^{(16)}$ recomiendan el uso de RP en el proceso enseñanza-aprendizaje del cuidado de enfermería, pues posibilita el trabajo en equipo y el intercambio de aprendizaje entre los involucrados.

En cuanto a las dimensiones evaluadas en la MIRS, la "presentación" del estudiante durante la entrevista clínica obtuvo los mayores puntajes en PS y RP. Esta dimensión considera la presentación, explicación del rol a desempeñar, y exploración de cómo dirigirse al paciente, lo cual favorece la comunicación efectiva en la relación de cuidado. La comunicación e interacción efectiva son necesarias en la relación de cuidado, aunque estudios revelan que la mayoría de las enfermeras perciben una falta de preparación en estas habilidades blandas ${ }^{(17)}$.

Los estudiantes también destacaron las dimensiones "uso de lenguaje técnico" y "técnicas de facilitación no verbal”, lo cual promueve una adecuada comunicación verbal y no verbal con el paciente. En esta última se encuentra el contacto visual, el lenguaje corporal, las expresiones faciales, y el contacto físico, entre otros. Indudablemente estas características contribuyen a establecer una relación terapéutica efectiva, permitiendo un cuidado de calidad. Los discursos manifiestan los beneficios percibidos en ambas metodologías, y también las dificultades que presentan, especialmente, durante la entrevista clínica con PS, desde la presentación hasta el término del encuentro. A pesar de ello, posicionan a la SC con PS como la estrategia que les permite transitar con mayor facilidad a expertos, dado el realismo experimentado. Aunque es más complejo y estresante, la presentación y el uso de técnicas verbales y no verbales, sugieren que la SC con PS contribuye mayormente en la transición de su aprendizaje. Esto podría explicarse en el contexto de lo señalado por Castro ${ }^{(18)}$, pues el hecho de que sean pacientes estandarizados genera confianza en el estudiante, por sobre el miedo e inseguridad que podría generar una primera entrevista a un paciente real. Estudios demuestran lo anterior ${ }^{(14)}$, señalando que las simulaciones minimizan el miedo a los procedimientos clínicos con pacientes reales, especialmente en el examen físico y en la comunicación durante la entrevista.

Los análisis cualitativos reflejan que los estudiantes perciben ambas simulaciones como efectivas en el desarrollo y aplicación de habilidades de comunicación. Dichos resultados destacan la realidad experimentada en ambas estrategias metodológicas, indicando algunas semejanzas y distinciones. Castro $^{(18)}$ comparte lo anterior, al señalar que la percepción de estudiantes sobre la SC realza los aspectos facilitadores del proceso de aprendizaje, concluyendo que las actividades de simulación son una experiencia de aprendizaje significativa y útil en el desarrollo de habilidades comunicativas, indicando satisfacción en el uso de ella ${ }^{(19)}$. Estas habilidades se convierten en competencias valiosas e insustituibles en todo profesional de la salud, pues tal como indica Chandawarkar ${ }^{(20)}$, el mejor entrenamiento quirúrgico sería ineficaz sin el desarrollo de habilidades de comunicación. Existen variados estudios que relacionan positivamente estas metodologías con el desarrollo de habilidades de comunicación en estudiantes y profesionales de la salud ${ }^{(8,12,19,21,22)}$, posicionando a la SC como una estrategia de enseñanza y aprendizaje efectiva ${ }^{(23)}$.

Otros autores como Henriksen et al. ${ }^{(24)}$ indican que la SC posee un enfoque que mejora la seguridad y calidad de la prestación de servicios de salud, siendo estos factores centrales en los discursos del actual estudio. Esto es semejante a lo descrito por Sebold et $\mathrm{al}^{(25)}$, al demostrar que en la relación profesional-paciente las habilidades sociales del profesional, la amabilidad, empatía y buena comunicación, están asociadas a la satisfacción y calidad de la atención.

Finalmente, las cuestiones sustanciales y repetitivas en los discursos sobre SC son las siguientes: percepción de estrés al ser observados, aportes en la toma de decisiones y en la resolutividad, y la promoción de un lenguaje apropiado en la 
relación de cuidado. Un estudio indica que la SC se acompaña de una experiencia de estrés por la demanda de una actuación rápida, la evaluación de las necesidades de salud en la persona, y la conformación de un equipo de salud ${ }^{(26)}$, sumado al escrutinio del evaluador y a la observación de sus pares. Asimismo, la contribución en la toma de decisiones y resolutividad ha sido referido por otros autores como aspectos positivos que acompañan el aprendizaje alcanzado por medio de la $\mathrm{SC}^{(27,28)}$. Lo anterior se comprende en la complejidad de las situaciones de salud y cuidado que debe enfrentar un enfermero(a), el cual lo emplaza a la integración de un conjunto de conocimientos, habilidades y actitudes en su práctica diaria ${ }^{(29)}$, permitiendo su transición a un estado de experto.

Los resultados de este estudio sugieren la necesidad de incorporar estas metodologías en el proceso formativo de enfermeros(as), quienes deben transitar de principiante a experto en el saber, saber ser y saber hacer ${ }^{(5,7)}$. Sin duda estos resultados podrían hallar significado en otros niveles o carreras, las cuales necesitan aplicar habilidades comunicativas para enfrentar una entrevista clínica efectiva y de calidad. Por ello es necesario estudiarlos más ampliamente, enfrentando las limitaciones del actual estudio: criterios de inclusión restringidos a una asignatura específica, lo cual limita el número de participantes, inexistencia de una medición previa que permita comparar el progreso de aprendizaje, y evaluación de un reducido número de dimensiones.

\section{CONCLUSIONES}

La aplicación de habilidades de comunicación durante la entrevista clínica fue evaluada positivamente desde la dimensión cuantitativa y cualitativa; obteniendo puntuaciones que demuestran un alto porcentaje de cumplimiento, y una evaluación perceptiva positiva entre los participantes. Aun con esto, se distingue una mejor aplicación de dichas habilidades en los contextos de SC con PS, siendo esta estrategia valorada con mayor realismo entre los participantes, lo cual les permite transitar con mayor facilidad desde un estado de principiante a otro de experto. Los participantes proponen que esta transición puede ser fortalecida al incorporar el $R P$ como una estrategia metodológica previa y preparatoria al PS; es decir en etapas iniciales del aprendizaje con entrevista clínica, permitiéndoles familiarizarse con la estructura de la entrevista, ganar confianza y seguridad.

Por lo anterior, es necesario diversificar las metodologías de enseńanza-aprendizaje aplicadas en el proceso formativo de enfermeros(as), teniendo presente las competencias que demandará la práctica clínica, especialmente en el ámbito de las habilidades de comunicación, las cuales son fundamentales en un cuidado efectivo y de calidad.

\section{REFERENCIAS}

1. Verano D, González S, Bolívar A, Fernández M, Galván I. Valoración de la competencia de comunicación oral de estudiantes universitarios a través de una rúbrica fiable y válida. Rev Bras Educ [Internet]. 2016 [citado 22 mar 2019]; 21(64): 39-60. Disponible en: https://www.scielo.br/pdf/ rbedu/v21 n64/1413-2478-rbedu-21-64-0039.pdf

2. Landete L. La comunicación, pieza clave en enfermería. Enfermería Dermatológica [Internet]. 2012 [citado 30 mar 2019]; 16: 16-19. Disponible en: https://dialnet.unirioja.es/servlet/articulo? codigo $=4069152$

3. Dos Reis M, Da Silva N, Pedroso B, Dutra A, Alvarez A, Da Cunha G. Simulação realística como ferramenta de ensino na comunicaçấo de situação crítica em cuidados paliativos. Esc Anna Nery [Internet]. 2020 [citado 28 may 2020]; 24(3): e20190271. Disponible en: https://doi. org/10.1590/2177-9465-EAN-2019-0271

4. Martínez T. Evaluación de la calidad de la atención de enfermería en la Clínica Internacional Trinidad. Rev Cubana Enfermer [Internet]. 2016 [citado 14 may 2019]; 32(2): 196-206. Disponible en: http:// www.revenfermeria.sld.cu/index.php/enf/article/ view/817

5. Molina P, Jara P. El saber práctico en Enfermería. Rev Cubana Enferm [Internet]. 2010 [citado 2 may 2021]; 26(2): 37-43. Disponible en: http:// scielo.sld.cu/pdf/enf/v26n2/enf05210.pdf

6. Benner P. Using the Dreyfus Model of Skill Acquisition to Describe and Interpret Skill Acquisition and Clinical Judgment in Nursing Practice and Education. Bulletin of Science, Technology \& Society [Internet]. 2004 [citado 2 may 2021]; 24(3): 188-199. Disponible en: $10.1177 / 0270467604265061$

7. Escobar B, Jara P. Filosofía de Patricia Benner, aplicación en la formación de enfermería: propuestas de estrategias de aprendizaje. Educación 
[Internet]. 2019 [citado 2 may 2021]; 28(54): 182202. Disponible en: https://dx.doi.org/10.18800/ educacion.201901.009

8. Villca S. Simulación clínica y seguridad de los pacientes en la educación médica. Rev Cien Tec In [Internet]. 2018 [citado 20 ago 2019]; 16(18): 7588. Disponible en: http://www.scielo.org.bo/pdf/ rcti/v16n18/a07v16n18.pdf

9. Urra E, Sandoval S, Irribarren F. El desafío y futuro de la simulación como estrategia de enseñanza en enfermería. Inv Ed Med [Internet]. 2017 [citado 24 may 2019]; 6(22): 119-125. Disponible en: https://doi.org/10.1016/j.riem.2017.01.147

10. Cleland JA, Abe K, Rethans JJ. The use of simulated patients in medical education: AMEE Guide No 42. Med Teach [Internet]. 2009 [cited $22 \mathrm{abr}$ 2019]; 31(6): 477-86. Disponible en: https://doi. org/10.1080/01421590903002821

11. Altamirano J. La simulación clínica: Un aporte para la enseñanza y aprendizaje en el área de obstetricia. Revista Electrónica Educare [Internet]. 2019 [citado 24 jun 2020]; 23(2): 167-187. Disponible en: doi: http://dx.doi.org/10.15359/ree.23-2.9

12. Frallicciardi A, Lotterman S, Ledford M, Prenovitz I, Van Meter R, Kuo C-L, et al. Training for Failure: A Simulation Program for Emergency Medicine Residents to Improve Communication Skills in Service Recovery. AEM Educ Train [Internet]. 2018 [citado 29 mar 2020]; 2(4): 277-87. Disponible en: https://doi.org/10.1002/aet2.10116

13. Centro de Habilidades clínicas. MIRS 2012, Master Interview Rating Scale versión en español [Internet]. Santiago, Chile: Facultad de Medicina, Universidad de Chile. 2015 Oct 15 [citado 20 oct 2020]; [6 pantallas]. Disponible en: https:// docplayer.es/95930407-Mirs-2012-masterinterview-rating-scale-version-en-espanol-centrode-habilidades-clinicas-facultad-de-medicinauniversidad-de-chile.html

14. Negri E, Mazzo A, Amado J, Pereira G, Dos Santos R, Pedersoli C. Clinical simulation with dramatization: gains perceived by students and health professionals. Rev Lat Am Enfermagem [Internet]. 2017 [citado 18 may 2019]; 25: e2916. Disponible en: https://doi.org/10.1590/15188345.1807.2916

15. Taylor S, Haywood M, Shulruf B. Comparison of effect between simulated patient clinical skill training and student role play on objective structured clinical examination performance outcomes for medical students in Australia. J Educ Eval Health Prof [Internet]. 2019 [citado 24 jun 2020]; 16:3. Disponible en: https://doi. org/10.3352/jeehp.2019.16.3

16. Sebold LF, Boell JEW, Fermo VC, Girondi JBR,
Santos JLG. Role-playing: teaching strategy that encourages reflections on nursing care. Rev Bras Enferm [Internet]. 2018 [citado 24 jun 2020]; 71(Suppl 6): 2706-12. Disponible en: http:// dx.doi.org/10.1590/0034-7167-2017-0733

17. Liebrecht C, Montenery S. Use of Simulated Psychosocial Role-Playing to Enhance Nursing Students' Development of Soft Skills. Creat Nurs [Internet]. 2016 [citado 8 mar 2019]; 22(3): 171175. Disponible en: https://pubmed.ncbi.nlm.nih. gov/29195526/

18. Castro R. Ficha didáctica: La simulación de entrevista clínica como estrategia innovadora para el fortalecimiento de habilidades comunicacionales de estudiantes de kinesiología. Revista Electrónica Diálogos Educativos [Internet]. 2017 [citado 15 dic 2019]; 33(18): 49-57. Disponible en: https://dialnet.unirioja.es/servlet/articulo?codigo= 6212472

19. Lermanda C, Muñoz J. Desarrollo precoz de habilidades de comunicación para realizar entrevistas clínicas mediante pacientes simulados estandarizados. Rev Educ Cienc Salud [Internet]. 2010 [citado 20 abr 2020]; 7(2): 134-140. Disponible en: http://www.udec.cl/ofem/recs/ anteriores/vol722010/artinv7210d.pdf

20. Chandawarkar R, Ruscher K, Krajewski A, Garg M, Pfeiffer C, Singh R, et al. Pretraining and posttraining assessment of residents' performance in the fourth accreditation council for graduate medical education competency: patient communication skills. Arch Surg [Internet]. 2011 [citado 10 abr 2019]; 146(8): 916-21. Disponible en: https://pubmed.ncbi.nlm.nih.gov/21844435/

21. Yuan Y, Scott S, Van Horn N, Oke O, Okada P. Objective Evaluation of a Simulation Course for Residents in the Pediatric Emergency Medicine Department: Breaking Bad News. Cureus [Internet]. 2019 Ene 16 [cited 13 ago 2020]; 11(1): e3903. Disponible en: https://pubmed.ncbi. nlm.nih.gov/30911458/

22. Hawkins A, Tredgett K. Use of high-fidelity simulation to improve communication skills regarding death and dying: a qualitative study. BMJ Support Palliat Care [Internet]. 2016 Dic [cited 8 may 2019]; 6(4): 474-478. Disponible en: https:// pubmed.ncbi.nlm.nih.gov/27316636/

23. Costa R, Medeiros S, Martins J, Coutinho V, Araújo M. Eficacia de la simulación en la enseñanza de inmunización en la enfermería: ensayo clínico aleatorio. Rev Lat Am Enfermagem [Internet]. 2020 [citado 10 sep 2020]; 28: e3305. Disponible en: https://doi.org/10.1590/1518-8345.3147.3305

24. Henriksen K, Rodrick D, Grace EN, Brady PJ. Challenges in Health Care Simulation: Are We 
Learning Anything New? Acad Med [Internet]. 2018 May [cited 10 jul 2020]; 93(5): 705-708. Disponible en: https://pubmed.ncbi.nlm.nih.gov/ 28817431/

25. Sebold L, Boell J, Fermo V, Girondi J, Santos J. Role-playing: estratégia de ensino que propicia reflexões sobre o cuidado de enfermagem. Rev Bras Enferm [Internet]. 2018 [citado 21 oct 2020]; 71(Suppl 6): 2706-2712. Disponible en: https:// doi.org/10.1590/0034-7167-2017-0733

26. Monti L, Dos Santos J, Del'Angelo N, Villela J, Domingues A, Dias $\mathrm{V}$, et al. Interdisciplinary simulation scenario in nursing education: Humanized childbirth and birth. Rev Lat Am Enfermagem [Internet]. 2020 [cited 20 jul 2019]; 28: e3286. Disponible en: https://doi. org/10.1590/1518-8345.3681.3286

27. Lavoie P, Micahud C, Belisle M, Boyer L, Gosslein E, Grondin M, et al. Learning theories and tools for the assessment of core nursing competencies in simulation: a theoretical review. J Adv Nurs [Internet]. 2018 [citado 9 may 2019]; 74(2): 23950. Disponible en: https://pubmed.ncbi.nlm.nih. gov/28815750/

28. Daley KM, Campbell SH. Integrating simulationfocused pedagogy into curriculum [Internet]. $3^{\mathrm{a}} \mathrm{ed}$. New York: Springer Publishing. 2018 [citado 8 may 2019]. Disponible en: https://www.researchgate. net/publication/330387355_Framework_for_ Simulation_Learning_in_Nursing_Education_ Making_It_Real

29. Shin H, Ma H, Park J, Ji E, Kim D. The effect of simulation courseware on critical thinking in undergraduate nursing students: multi-site prepost study. Nurse Educ Today [Internet]. 2015 [citado 18 may 2019]; 35(4): 537-42. Disponible en: https://pubmed.ncbi.nlm.nih.gov/25549985/ 